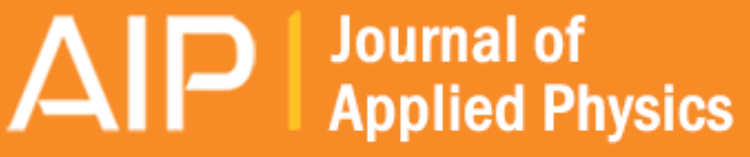

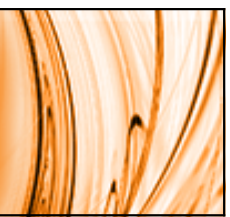

Limitations to laser machining of silicon using femtosecond micro-Bessel beams in the infrared

David Grojo, Alexandros Mouskeftaras, Philippe Delaporte, and Shuting Lei

Citation: Journal of Applied Physics 117, 153105 (2015); doi: 10.1063/1.4918669

View online: http://dx.doi.org/10.1063/1.4918669

View Table of Contents: http://scitation.aip.org/content/aip/journal/jap/117/15?ver=pdfcov

Published by the AIP Publishing

\section{Articles you may be interested in}

Interference rings formation inside cellulose from a back-reflected femtosecond laser pulse

J. Appl. Phys. 112, 066101 (2012); 10.1063/1.4751359

Micromachining along a curve: Femtosecond laser micromachining of curved profiles in diamond and silicon using accelerating beams

Appl. Phys. Lett. 101, 071110 (2012); 10.1063/1.4745925

Study of laser beam propagation in microholes and the effect on femtosecond laser micromachining

J. Appl. Phys. 109, 123506 (2011); 10.1063/1.3595671

Ablation and cutting of silicon wafer and micro-mold fabrication using femtosecond laser pulses

J. Laser Appl. 19, 240 (2007); 10.2351/1.2795754

Efficiency of silicon micromachining by femtosecond laser pulses in ambient air

J. Appl. Phys. 99, 083101 (2006); 10.1063/1.2187196

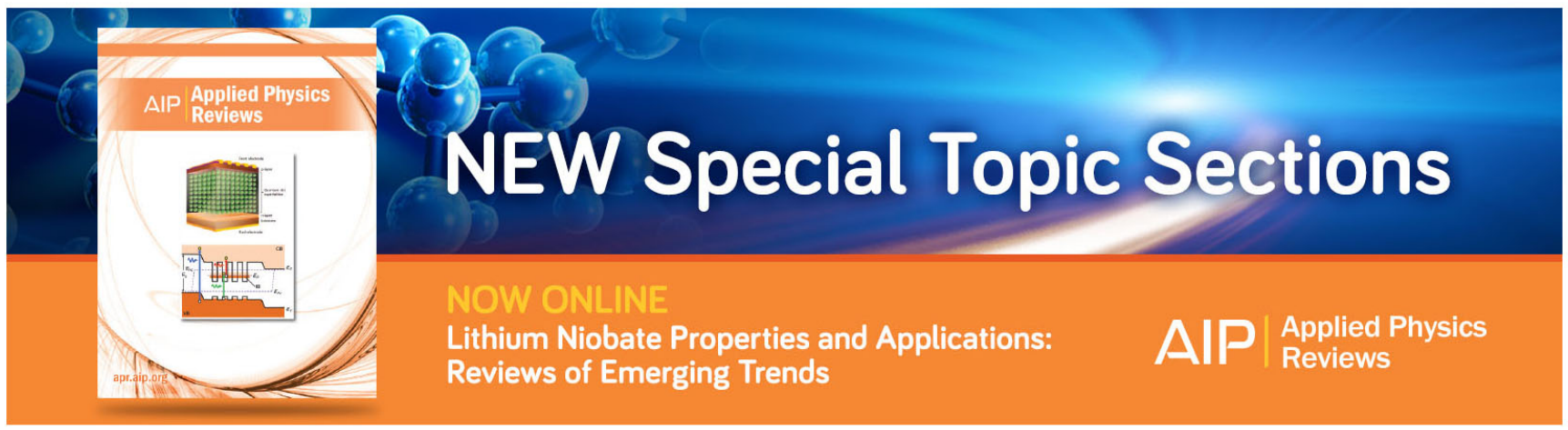




\title{
Limitations to laser machining of silicon using femtosecond micro-Bessel beams in the infrared
}

\author{
David Grojo, ${ }^{1, a)}$ Alexandros Mouskeftaras, ${ }^{1}$ Philippe Delaporte, ${ }^{1}$ and Shuting Lei ${ }^{2}$ \\ ${ }^{1}$ Aix-Marseille University, CNRS, LP3 UMR 7341, F-13288 Marseille, France \\ ${ }^{2}$ Industrial and Manufacturing Systems Engineering, Kansas State University, Manhattan, Kansas 66506, USA
}

(Received 12 February 2015; accepted 9 April 2015; published online 20 April 2015)

\begin{abstract}
We produce and characterize high-angle femtosecond Bessel beams at 1300-nm wavelength leading to nonlinearly ionized plasma micro-channels in both glass and silicon. With microjoule pulse energy, we demonstrate controlled through-modifications in $150-\mu \mathrm{m}$ glass substrates. In silicon, strong two-photon absorption leads to larger damages at the front surface but also a clamping of the intensity inside the bulk at a level of $\approx 4 \times 10^{11} \mathrm{~W} \mathrm{~cm}^{-2}$ which is below the threshold for volume and rear surface modification. We show that the intensity clamping is associated with a strong degradation of the Bessel-like profile. The observations highlight that the inherent limitation to ultrafast energy deposition inside semiconductors with Gaussian focusing [Mouskeftaras et al., Appl. Phys. Lett. 105, 191103 (2014)] applies also for high-angle Bessel beams. (C) 2015 AIP Publishing LLC. [http://dx.doi.org/10.1063/1.4918669]
\end{abstract}

\section{INTRODUCTION}

Focused femtosecond laser beams have shown their ability for local modification in any type of transparent dielectric materials. This has opened a wide range of $3 \mathrm{D}$ microfabrication technologies with subsequent applications in micro-optics, micro-fluidics, and nanosurgery. ${ }^{1}$ However, there is today no counterpart in narrow gap semiconductors because they are opaque at the fundamental wavelength of commonly used femtosecond lasers (e.g., Ti:sapphire). In this context, new emerging femtosecond fiber lasers emitting in the short-wave infrared (SWIR) region of the spectrum (1.1-2.5 $\mu \mathrm{m})$ may represent highly attractive tools (e.g., Erbium doped) to extend the technologies to silicon.

Nevertheless, first SWIR femtosecond laser experiments in semiconductors reveal that the intrinsic properties of narrow gap materials prevent the occurrence of confined breakdown in the bulk with conventional Gaussian focusing interaction arrangements. ${ }^{2-5}$ This is caused by a clamping of the intensity at a level far below the required threshold for modifications. Such a specificity likely relies on nonlinear Kerr-induced phase distortions and nonlinear beam depletion in the prefocal region due to, respectively, the high thirdorder nonlinearity and the high multiphoton absorption cross-section of these materials. ${ }^{6,7}$ Practically, attempts to increase the focus intensity beyond the clamped value by simply increasing the input energy fail and result in an increase of the overall interaction volume prior to the focus within which nonlinear energy deposition occurs. ${ }^{5}$ For the highest pulse energies, we also expect temporal splitting and spatial breakup of the beam according to reports on high intensity filamentation experiments in various medium (e.g., Ref. 8).

In Bessel beams, the energy is everywhere directed at a given angle toward the optical axis providing two advantages

${ }^{\text {a)} E l e c t r o n i c ~ m a i l: ~ g r o j o @ l p 3 . u n i v-m r s . f r ~}$ for controlled interactions in the bulk of highly refractive materials. First, it makes the interactions free from spherical aberrations in comparison with focused Gaussian beams that contain a wide range of incidence angles. Second, an even more important feature is that their propagation strongly resists nonlinear distortions because intensities remain modest until the central core region is reached. It is only here that significant nonlinear effects (including nonlinear beam distortions and nonlinear absorption) can occur. ${ }^{9}$ Bessel beams therefore intrinsically allow controlled interactions at higher intensities compared to Gaussian beams. The benefit is even more pronounced at increased Bessel angles. This has been already exploited for fabricating high-aspect ratio $(>100)$ nanochannels in glassy materials using single femtosecond pulses at fundamental wavelengths. ${ }^{10}$

Taking all considerations together, it raises the fundamental question: Do the advantages of high-angle Bessel beams lead to a situation in which intensity clamping for SWIR wavelengths in semiconductors can be overridden so that bulk modification can occur? The answer to this question may offer new solutions for high aspect-ratio machining applications and could change the way $3 \mathrm{D}$ microdevices are fabricated inside silicon chips.

In this paper, we investigate the interaction of highintensity femtosecond micro-Bessel beams at 1300-nm wavelength inside samples of silicon and borosilicate (BS) glass (for comparison). This is achieved by conical lens (axicon) focusing and an experimental arrangement to magnify the angle of the waves contributing to the Bessel beams. We study the impact of the beams on the materials and analyze how the interactions act on the beam by high-resolution beam profile analysis. We show that well-defined micro-channels are formed in glass. However, despite the steep angular components forming our beam $\left(>20^{\circ}\right)$, we measure that the clamping of the intensity, the subsequent free-carrier density and absorbed energy remain at levels far below the thresholds for local modification in bulk silicon. There is today a general 
trend toward ultrashort pulses for ultraprecision micromachining applications but our investigations with SWIR femtosecond Bessel beams suggest that only longer pulses ${ }^{11,12}$ or multiple pulses ${ }^{13}$ can be envisioned for deep micromachining inside silicon.

\section{EXPERIMENTAL SETUP AND METHODS}

Our experiments use the setup shown in Fig. 1. The beam from an amplified Ti:Sapphire femtosecond laser system at $800-n m$ wavelength (Spectra-Physics, Hurricane) and $1-\mathrm{kHz}$ repetition rate is directed into an optical parametric amplifier (OPA) operated to produce signal and idler beams with respective wavelengths: $1300 \mathrm{~nm}$ and $2100 \mathrm{~nm}$. A long pass filter with wavelength cut-off at $850 \mathrm{~nm}$ eliminates any residual pump or visible light from the OPA. Then, the beams are spatially separated using a short pass dichroic mirror with cutoff wavelength at $1500 \mathrm{~nm}$. An additional broadband high contrast nanoparticle polarizer is used to eliminate any residual idler beam, which is orthogonally polarized to the signal beam used for the experiments. The combination of a half-waveplate and polarizer allows variable attenuation of the beam with a maximum energy of $12 \mu \mathrm{J}$ per pulse hitting the target materials. The duration of the pulses is $110 \mathrm{fs}$ (FWHM) as measured using a single-shot infrared autocorrelator (Light Conversion, TiPA AT5C3).

To produce a high-angle Bessel beam at $1300 \mathrm{~nm}$, we implement an axicon based method. ${ }^{14}$ To avoid non-linear effects and/or the occurrence of damage due to intense beam propagation in optical elements, we use first a thin conical lens of refractive index 1.45 (anti-refection coated) with only $1^{\circ}$ base angle (Thorlabs, AX251-C). When our collimated Gaussian beam is incident on the axicon, the different

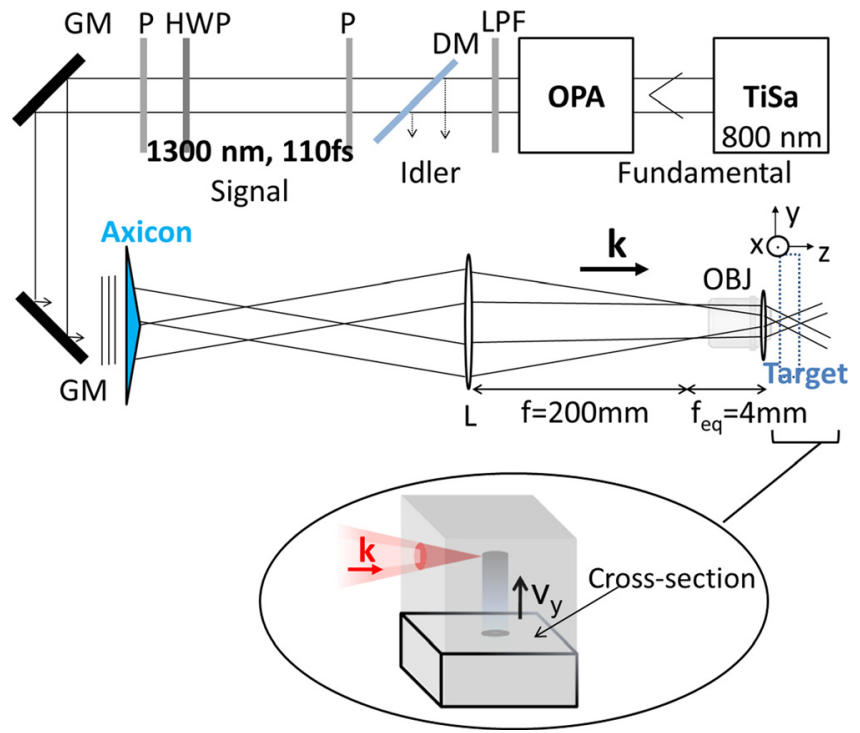

FIG. 1. Setup to produce SWIR femtosecond Bessel beams for micromachining experiments. TiSa is a Ti:sapphire femtosecond laser operated at $1 \mathrm{kHz}$; OPA is an optical parametric amplifier; LPF, DM, and P are, respectively, a long pass filter, a dichroic mirror, and a polarizer to select the 1300$\mathrm{nm}$ signal beam from the OPA; HWP is a half wave plate; and GM are gold mirrors. The micro-Bessel beam shaping is obtained using a lens (L) and a microscope objective $(\mathrm{OBJ})$ in a 4-f arrangement for $\times 1 / 50$ demagnification of the Bessel beam formed with a low angle axicon. portions of the beam deviate towards the optical axis with an angle of $0.45^{\circ}$ and resulting, by interference, in a Bessel-like laser energy distribution with a spot size of $\approx 60 \mu \mathrm{m}$. The length along the optical axis exceeds $250 \mathrm{~mm}$ (FWHM) in air as observed by direct beam characterization using a camera behind the axicon. The generated Bessel beam is then de-magnified by a factor of 50 using the 4-f image relay geometry shown in Fig. 1. The 4-f arrangement consists of a plano-convex lens of focal length $200 \mathrm{~mm}$ and a long working distance microscope objective lens (50X/NA0.42/NIR, Mitutoyo) with an effective focal length of $4 \mathrm{~mm}$. This forms a micro-Bessel beam based on waves making an angle of $22.5^{\circ}$ with respect to the optical axis. This is a very highangle that corresponds to the effect of a virtual axicon of $\approx 36^{\circ}$ base angle. The latter would represent a very thick optical element and is not available commercially. It is also worth mentioning that there is a limit above which single axicons cannot operate due to total internal reflection $\left(\approx 42^{\circ}\right)$ but the limit does not apply with the image relay arrangement. Even if it were practically feasible to generate angles steeper than $22.5^{\circ}$ with our setup, there is no motivation for a silicon micromachining experiment because s- and ppolarized components found in a focused beam geometry would exhibit different Fresnel reflection coefficients at the front interface, ${ }^{15}$ leading to distortion of the beam focus inside the material. As described in Sec. III, the femtosecond micro-Bessel beam generated with this setup is fully characterized before material modification experiments.

The micro-Bessel focus is then used for the machining of embedded modifications inside double-side polished BS glass (microscopy cover slips) and silicon samples with different thicknesses. All tested silicon substrates are 100orientation, high-resistivity crystals (intrinsic or $n$-doped substrates with $R>10 \Omega \mathrm{cm}$ ) so that all are fully transparent to the laser wavelength and we avoid any avalanche effects from equilibrium carrier concentration. ${ }^{16}$ Cross-sectioning of the substrates is performed to observe the damage in the bulk of materials. However, because it would be very challenging to cut a substrate in the middle of a micro-sized region, we decided to apply a conventional scanning procedure to produce extended modifications by translating the sample using a motorized X-Y micropositioning stages at $0.1 \mathrm{~mm} / \mathrm{s}$ perpendicular to the propagation direction of the laser beam (see inset in Fig. 1). Accordingly to the $1 \mathrm{kHz}$ repetition rate of our laser, any exposed region experiences a few tens of shots depending on the spot size (in fact, 100 (respectively, 10) shots for a $10-\mu \mathrm{m}$ (respectively, $1-\mu \mathrm{m})$ spot size). Then, we vary the laser pulse energy and the focusing depth ( $Z$ position) when writing the modifications. In all material characterizations for this paper, the laser light is polarized perpendicularly to the writing direction but we checked that a polarization parallel to the writing direction leads to very similar observations. After laser irradiations, the sample is cut (or cleaved for $\mathrm{Si}$ ) in half, normal to the writing direction. The two inside surfaces are directly characterized (without cleaning or polishing). Cross-sectional images of the modified regions are obtained using a reflected light optical microscope in bright-field configuration and a scanning electron microscope (JEOL, JSM 6390). To avoid SEM 
imaging artifacts caused by surface charging, glass samples are coated with a $5 \mathrm{~nm}$ layer of Gold.

\section{RESULTS AND DISCUSSION}

\section{A. Beam profiling and 3D-imprint inside glass}

For the interpretation of material modification experiments, it is of importance to deal with a well-controlled beam profile that is fully characterized. There are two ways that can be used to characterize the intensity distribution of our micro-Bessel beam impinging our targets. The first is summarized in Fig. 2 and consists in beam profiling using a high-resolution imaging technique in air. The second is to perform an imprint of the laser energy in wide-band gap dielectrics so that the intensity distribution can be retrieved in the 3 dimensions from spatially resolved material analyses.

In Fig. 2(a), we sketch the arrangement used for direct imaging of the infrared femtosecond micro-Bessel beam. We use a long working distance microscope objective with a higher numerical aperture (100X/NA0.7/NIR, Mitutoyo) than that used to produce the Bessel-like focus. The objective, a tube lens $(f=200 \mathrm{~mm})$ and a high resolution silicon CCD camera (pixel size $=4.65 \mu \mathrm{m}$ ) are mounted on a precision motorized stage so that we can acquire section images of the beam separated by $2 \mu \mathrm{m}$ steps along the optical axis. From the collected stack of images, we reconstruct the full $3 \mathrm{D}$ intensity distribution in the micro-Bessel region. It is important to note that the images rely on a nonlinear response of the detector because silicon is fully transparent at the wavelength of our beam. Working with ultrashort pulses, we found that very modest pulse energies can generate a CCD signal $S_{C C D}$ based on a two-photon absorption response.
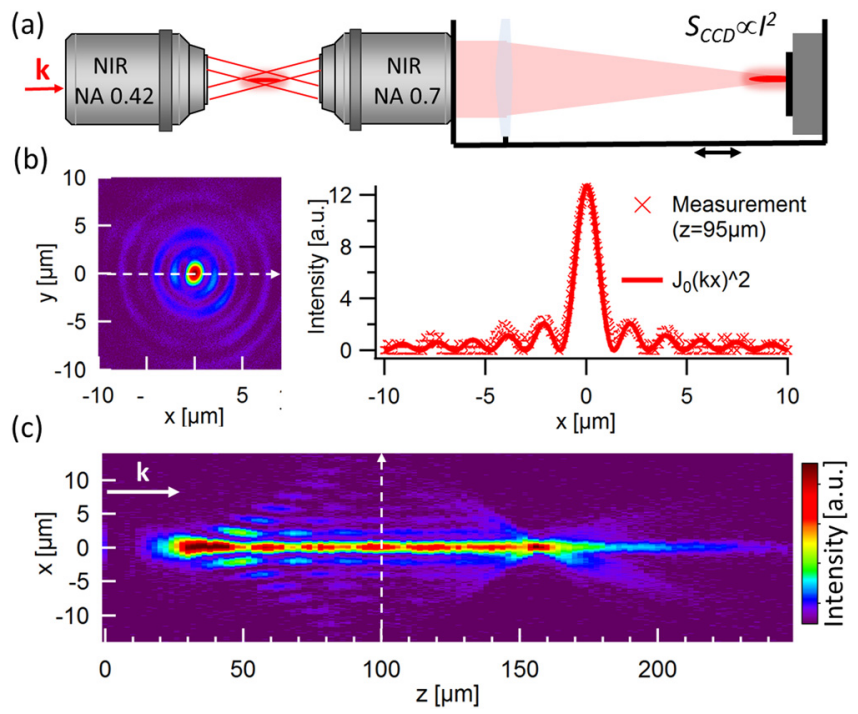

FIG. 2. Experimental characterization in air of the high-angle Bessel-like focus generated with femtosecond laser light at 1300-nm wavelength. (a) A microscopy system with $\times 100$ magnification is translated to acquire transverse beam profile images (b) at different distances along the optical axis. From the collected stack of images, we reconstruct the intensity distribution in the longitudinal direction (c). The reconstruction evidences the elongated focus with a transverse profile which compares favorably with a theoretical Bessel function (continuous red curve).
Using a calibration measurement (not shown here), we can demonstrate that it is possible to directly convert the image in intensity profile according to $I \propto \sqrt{S_{C C D}}$. The main advantage of the approach is that it avoids the use of infrared sensitive sensors (e.g., InGaAs) that offers a linear response and subsequently a better dynamic range for intensity measurements (see Sec. III C) but less pixel resolution which is the main relevant aspect here. Figure 2(b) shows a retrieved beam focus profile and a comparison in the radial direction to a zeroth-order Bessel function $J_{0}^{2}(k x)$. Interestingly, we note that the measurements give a very good agreement with the Bessel function as it was originally targeted with the design of our experiment. Also, it is worth mentioning that a central spot diameter of $1.25 \mu \mathrm{m}$ is measured (FWHM) which is smaller than the spot which would be achieved with Gaussian beam focusing using the same microscope objective $(\mathrm{NA}=0.42)$. Figure $2(\mathrm{c})$ shows the intensity reconstruction $I(x, z)$ in the longitudinal direction to directly indicate the extent of the Bessel-like beam focus. Despite some oscillations, the peak intensity is maintained close to its maximum along a distance exceeding $130 \mu \mathrm{m}$ in air. We note also a pronounced dissymmetry with a sharp rise $(\approx 10 \mu \mathrm{m})$ and a long tail $(>50 \mu \mathrm{m})$ ending the high intensity region.

We can also use the material modifications induced by our beam inside the materials to retrieve some spatial information about our beam in the conditions of the experiments. It is today widely demonstrated that material breakdown by intense laser pulse in transparent dielectrics can leave directly a well-defined imprint of the local intensity and also polarization under appropriate conditions. ${ }^{17-19}$ This relies on the threshold nature of the material response, the strong electric field, and the limited thermal effects in ultrafast regimes. The extremely high-aspect ratio nanostructures fabricated inside glass with femtosecond Bessel beams at fundamental wavelengths (e.g., $800 \mathrm{~nm})^{10}$ are a confirmation that it must represent a way to observe the energy distribution of our femtosecond micro-Bessel beam generated at 1300-nm wavelength. We can even expect an enhancement of the deterministic character ${ }^{20}$ in our experiment thanks to the increase of nonlinearity associated with the use of longer wavelengths. ${ }^{7}$ Figure 3 shows optical and scanning electron microscopy images of a cross-section of a borosilicate glass slide irradiated with pulses of $4.2 \mu \mathrm{J}$ at different depths (see Sec. II). This pulse energy is about twice the energy threshold for the observation of modifications so that we can consider the dimensions of the modified region to be approximately the dimensions of our beam at half-maximum intensity. For comparison, we display in the same figure (Fig. 3) the extrapolation (from the measurement in air) of the beam focus as if it were in glass. The extrapolation assumes no distortion by aberration and the total absence of non-linear propagation effects so that the intensity distribution is simply the measurement shown in Fig. 2 but stretched along the optical axis by multiplying the $z$ coordinate by the refractive index of the material $n_{0}=1.5$. Accordingly, we predict a focus length exceeding $195 \mu \mathrm{m}$ in glass that is longer than the thickness of the processed substrate. The red circles A and B show, respectively, the evolution of the front and back damage positions observed inside the material when the target position is moved 


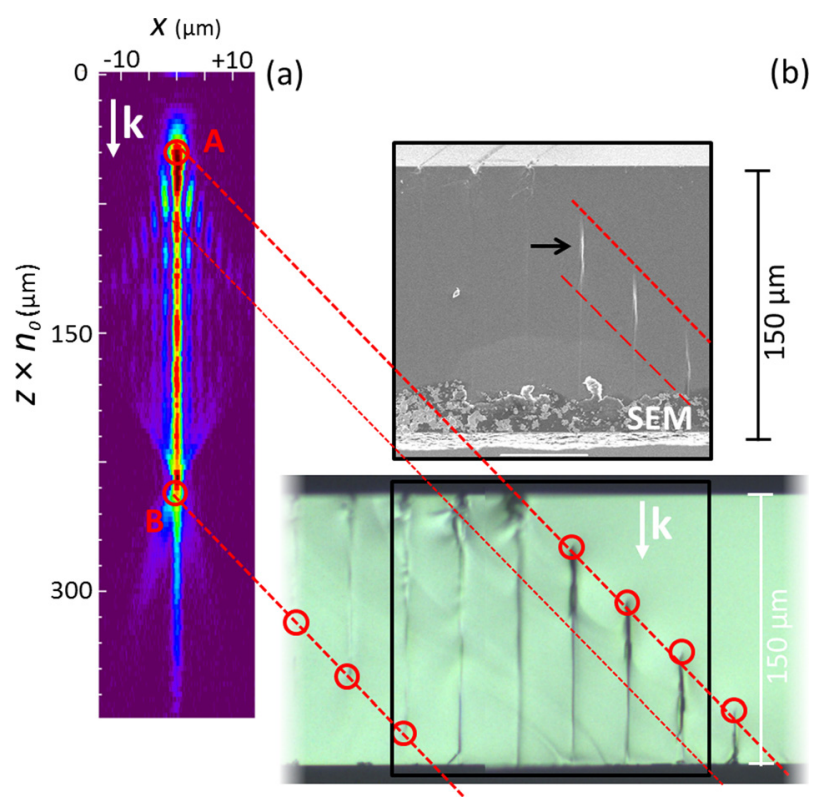

FIG. 3. Bessel-like focus imprinting inside glass. (a) Laser intensity distribution inside a material of refractive index 1.5 extrapolated from our measurement (Fig. 2) in the absence of nonlinear propagation and/or aberration. The length A-B ( $\cong 190 \mu \mathrm{m})$ of the high intensity channel matches that of the modifications written with a pulse energy of $4.2 \mu \mathrm{J}$ for different focusing depth and observed by optical (b-bottom) and electron microscopy (b-top). The arrow shows material disruptions in the region of maximum intensity.

by $20-\mu \mathrm{m}$ steps along the optical axis (depth). We note that the positions are shifted to about $30-\mu \mathrm{m}$ for each step accordingly to the refractive index of glass. Extrapolating the total length of the modifications, we see that there is a very good correspondence with the length of the expected Bessel focus (see dashed lines to guide eyes). It is also informative to stress that more pronounced damage are produced in the front part where we expect the highest intensity. The SEM images reveal only this front-region indicating likely that the occurrence of material disruptions ${ }^{21}$ and/or nanovoid formation ${ }^{22}$ in this volume. The excellent correspondence between the characterization in air and the morphology of the modifications in glass demonstrates the degree of control and knowledge that we have obtained on our shaped beam for the silicon micromachining experiment. It also confirms the robustness to aberration and potential distortions induced by non-linear propagation in modest refractive index materials such as borosilicate glass matrices.

\section{B. Comparison to silicon material response}

Due to the high permittivity of silicon, we expect that the beam energy will be distributed over a longer distance due to refraction at the front interface $\left(l_{S i}=l_{\text {air }} \times n_{S i}\right.$ $=450 \mu \mathrm{m})$. We predict also a higher Fresnel reflection coefficient $\left(R_{S i} \approx 31 \%\right)$ leading to more losses. If the Bessel intensity varies linearly with incident energy, the combination of both effects would lead to a typical decrease of the peak intensity by a factor of three compared to the same experiment performed in glass (where $l_{\text {glass }}=195 \mu \mathrm{m}$ and $R_{\text {glass }}=4 \%$ ). First, to somehow compensate, we choose to perform a comparison to silicon at pulse energy of $12 \mu \mathrm{J}$ about three-times higher than that used to obtain the modifications shown in Fig. 3. This represents the maximum energy for the present experiment and lower energies (down to $0.5 \mu \mathrm{J}$ ) were also investigated. Here, it is worth noting that this compensation is probably exaggerated because of the large two-photon absorption coefficient of silicon at $1300 \mathrm{~nm}$ $\left(\approx 1.5 \mathrm{~cm} / \mathrm{GW}\right.$ for $\left.\mathrm{Si}^{6,23}\right)$ compared to the higher order multiphoton absorption in glass. The compensation would be even exaggerated if we were comparing interactions with a similar nonlinear order in both materials because the two-photon absorption coefficient is on the order of $10^{-2} \mathrm{~cm} / \mathrm{GW}$ for fused silica and other glassy materials in the ultraviolet region of the spectrum. ${ }^{24,25}$

The direct comparison with the Bessel beam is shown in Figure 4 where SEM cross-section images of a glass substrate (a) and a 500- $\mu \mathrm{m}$ thick silicon substrate after the laser writing procedure with a pulse energy of $12 \mu \mathrm{J}$ are presented. Between lines, the target position (respectively, focus position) is moved by $20 \mu \mathrm{m}$ (respectively, $30 \mu \mathrm{m}$ ) along the optical axis for BS glass while it is moved by $10 \mu \mathrm{m}$ (respectively, $35 \mu \mathrm{m}$ ) for silicon. The dashed dotted lines show the evolution of the positioning (center) of the elongated focus to guide eyes. For glass, we confirm that this pulse energy allows complete through hole modification when the focus is appropriately positioned. ${ }^{10,26,27}$ This is of great importance for technological considerations, especially when ablation along the channel is achieved. It offers the possibility for direct microchannel drilling ${ }^{10,28}$ and ultrahigh precision cutting of transparent dielectrics. ${ }^{29,30}$ In silicon, the material response is drastically different. We note that we have enough energy density for surface damage. The stronger damages in comparison to glass are associated to the high two-photon absorption coefficient of Si. However, there is no noticeable material modification in the bulk observed by SEM or optical microscopy. In this experiment, the only way to increase the depth and by extension the aspect ratio of the induced modification is to increase the number of shots (not shown here) but we have not investigated this parameter because it was not relevant to this study concentrating on processes for direct energy deposition. Our conclusions for Bessel beams are the exact analog of the limitations reported in recent works concluding on the impossibility to damage bulk semiconductors with tightly focused Gaussian ultrafast lasers in the SWIR domain. ${ }^{3-5}$ To confirm the analogy, we have repeated the writing procedure removing the axicon and the $200 \mathrm{~mm}$ lens in the optical path (see Fig. 1) so that we perform the experiment with Gaussian focusing with NA similar to that used for the Bessel beam interactions. Then, the configuration is very similar to our previous works. ${ }^{5,7,31}$ The results are shown in Fig. 4(c) and the non-occurrence of material breakdown is confirmed. No confined subsurface modification could be achieved with energy up to $90 \mu \mathrm{J}$ and multiple pulse irradiations.

Among the differences between the experiments carried out in glass and in silicon, one is the material thickness. Most advanced technological demonstrations of high aspectratio glass ablation report on the need to ensure a breakdown point at an exit surface to facilitate material evacuation. ${ }^{10}$ Consequently, we decided to repeat the micromachining experiments for ultrathin silicon substrates. Figure 5 shows 


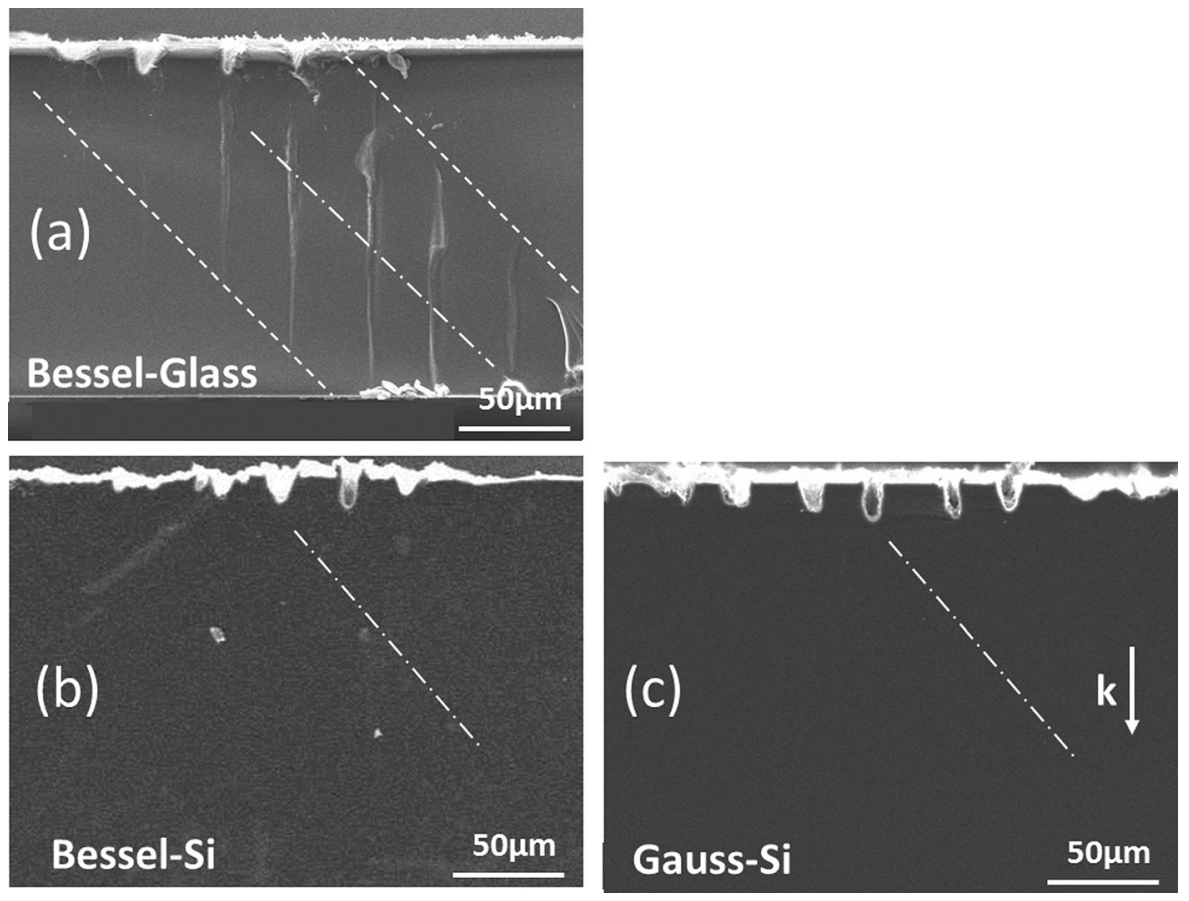

FIG. 4. Comparison between modifications induced in glass and silicon for a pulse energy of $12 \mu \mathrm{J}$ delivered on the target. For all substrates, lines are written at different depths and crosssections are observed. The material disruption induced by our Bessel beam in glass extends over the entire thickness (a) but remains confined at the front surface in Si. Similarly, there is no confined modification observed in the bulk when Gaussian focusing in applied (c). The dashed dotted line shows the evolution of focus position to guide the eyes.

the optical images of about $150 \mu \mathrm{m}$ thick glass and silicon. According to the beam characterization, we expect to be able to place the entire sample between both extremities of the micro-Bessel filaments A and B (shown in Fig. 3). However, the cross-sections in Figure 5 reveal that the bulk of silicon substrates remains in pristine condition for all tested substrate positions. This shows that the limitation observed is not directly connected with the material thickness or depth of processing.

\section{Intensity clamping and distortions of the Bessel profile inside silicon}

As already mentioned, this work is not the first to report on the incapacity of ultrafast SWIR laser pulses to modify the inside of silicon and other semiconductor materials.

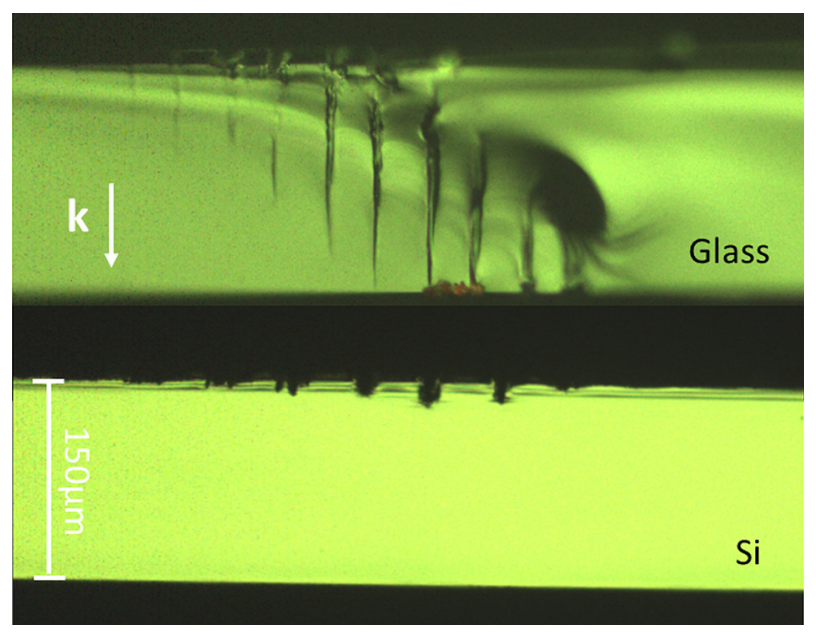

FIG. 5. Comparison of the material modifications induced with the same micro-Bessel beam $(E=11 \mu \mathrm{J})$ in the glass substrate and an ultra-thin silicon wafer. The written structures are prepared with the same procedure as previous figures (lines at different depths). Neither confined bulk damage nor back surface modification is found with ultra-thin silicon wafers.
Ultrafast pump and probe imaging of the induced microplas$\operatorname{mas}^{3,5}$ and detection of potential subsequent damages ${ }^{4}$ were recently employed to demonstrate this aspect even for the tight Gaussian focusing of pulses shorter than $150 \mathrm{fs}$. All studies indirectly conclude that the limitation relies on an inefficient beam delivery at the focus either resulting from pre-focal absorption or from light delocalization associated with nonlinear propagation effects. Here, we can provide a direct demonstration of this assumption in our Bessel optics experiment.

We cannot directly measure the beam profile inside the silicon using the same procedure as that used in Sec. III A in air because the acquired images would be affected by the nonlinear propagation of the beam in the silicon region between the imaged plane and the back of the substrate. To avoid this issue and make images in the real space, the microscopy system is adjusted to image the back surface of a 1-mm thick silicon substrate. Then, the beam focus is approached by translating the laser focusing objective $(\mathrm{NA}=0.42)$ for profile analyses in the middle of the micro-Bessel filament (between A and B shown in Fig. 3(a)). Because there is only air and no interaction in air between the image plane and the imaging objective input (confirmed by the intensity measured at $\approx 10^{11}$ $\mathrm{W} \mathrm{cm}{ }^{-2}$ hereafter), the procedure images the intensity distribution of a beam focus as if it were focused at a 1-mm depth below the surface. For these measurements, we replace the silicon CCD by an InGaAs array sensor (Raptor OWL 640) for an increased sensibility of the intensity measurement. By imaging a Gaussian beam focused in air in combination to a pulse energy measurement (powermeter OPHIR 3A), we can calibrate the measurement and report on absolute intensity profiles.

The results are shown in Fig. 6 where we plot the intensity response as a function of input pulse energy. For clarity, the measurement is shown here in linear scale but we can perform the measurement all over a dynamic range of $50 \mathrm{~dB}$ 


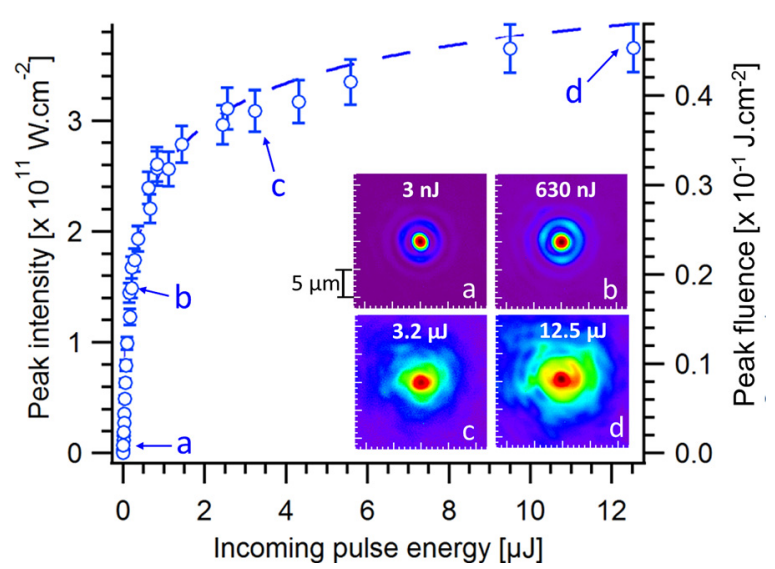

FIG. 6. Measurement of the peak intensity as a function of incoming pulse energy for our Bessel focus centered at the back surface of a 1-mm thick silicon substrate. Images of the beam profile at the back of the substrate are acquired using a highly sensitive InGaAs sensor for increasing laser pulse energies. The peak intensity is retrieved from the images according to a calibration of the camera response to a Gaussian beam focused in air. The profiles (a)-(d) are normalized to their maximum intensity and reveal how the Bessel profile is degraded with the clamping of the intensity.

with a constant relative uncertainty (using a set of calibrated neutral density filters). The curve shows unambiguously a saturation of the maximum intensity that can be delivered for these conditions. To reveal more details, we show four images of the beam profile at different pulse energies from $3 \mathrm{~nJ}$ to $12.5 \mu \mathrm{J}$. For $3 \mathrm{~nJ}$, we measure a sharp peak and a ring structure very similar to the Bessel-like beam measured in air (Fig. 2(b)). All images exhibit a sharp central peak, but we note the energy is progressively redistributed in favor of its surrounding region and the concentering ring structure is progressively degraded with the increase of energy. The measured profiles indicate that the Bessel characteristics (and potential benefits) are likely to completely vanish at the saturation intensity.

A major aspect here is the absolute measurement of the maximum intensity that can be achieved inside $\mathrm{Si}$ found at $3.7 \times 10^{11} \mathrm{~W} \mathrm{~cm}^{-2}$ and a corresponding peak fluence of $0.046 \mathrm{~J} \mathrm{~cm}^{-2}$ (Fig. 6, right axis). We already note that these values are below the known thresholds for surface modification of $\operatorname{silicon}^{32}\left(F_{t h} \approx 0.1 \mathrm{~J} \mathrm{~cm}^{-2}\right)$, which explains why back surface processing also cannot be envisioned with this beam. However, the intensity remains significantly above the threshold for two-photon ionization ${ }^{7}\left(10^{10} \mathrm{~W} \mathrm{~cm}^{-2}\right)$, and we can use the intensity measurement for estimates of excitation densities achieved in the bulk of silicon. For an estimate of the maximum free-carrier density, we can use the relation $N_{\text {max }}=\left(\tau \beta I_{\text {max }}^{2}\right) /(2 \hbar \omega)$ where $\tau, \beta$, and $\hbar \omega$ are, respectively, the pulse duration, the two-photon absorption coefficient, and the photon energy. This assumes that only two-photon absorption contributes to nonlinear ionization which is consistent with the absence of avalanche signatures for similar experimental conditions. ${ }^{16}$ Using a two-photon absorption coefficient $^{6,23,33}$ in the range of $0.5-1.5 \mathrm{~cm} / \mathrm{GW}$, we find a maximum free-carrier density $N_{\max }=[2.9-8.1] \times 10^{19}$ $\mathrm{cm}^{-3}$ which is in remarkable agreement with our previous measurements based on ultrafast imaging of the plasma. ${ }^{5}$ Assuming that all the absorbed energy to create the plasma is transferred to the lattice, the material can suffer a corresponding energy density $E_{a b s}=2 \hbar \omega N_{\max }$ in the range of $8.8-16.8 \mathrm{~J} / \mathrm{cm}^{3}$, which is far below the energy density of $\approx 6 \mathrm{~kJ} \mathrm{~cm}^{-3}$ required to heat silicon to the melting point and overcome the latent heat. ${ }^{34}$ This is consistent with the absence of modification.

\section{SUMMARY AND PERSPECTIVES}

In conclusion, there is an inherent intensity clamping occurring inside silicon in two-photon ionization initiated ultrafast laser interactions. So far, this was reported for tightly focused infrared femtosecond Gaussian beams. Here, we have designed and performed an experiment involving beam shaping to a Bessel-like beam for which the maximum clamping intensity is expectedly increased beyond the value for a Gaussian beam. ${ }^{9}$ The well-defined through modifications that we have achieved in glass with our beam evidence the good control that we could obtain with the formation of high-angle Bessel-like beam. However, using micro-Joule energy pulses, we have measured that the intensity delivered remains below $10^{12} \mathrm{~W} \mathrm{~cm}^{-2}$ in silicon which confirms that the absence of modification is also directly connected to an inefficient beam delivery.

While detailed nonlinear propagation simulations are required to further elucidate the origin of beam distortions found in the beam profile, the subsistence of a sharp central peak for the entire energy range tested encourage us to extend the study at higher beam energies. The use of a microscope objective in these experiments limits the maximum energy that could be envisioned to create the microBessel beam. In future works, we plan to repeat the study by using a high-energy OPA available in our laboratory (LP3ASUR facility) and by designing an experiment supporting pulse energies up to $1 \mathrm{~mJ}$ at $1300 \mathrm{~nm}$. One objective will be to determine whether one can break the limitation and lead to silicon modifications.

It is worth noticing that the limitation in the energy deposition is a specificity of the femtosecond regime, and it is now possible to demonstrate modifications initiated by twophoton ionization by simply using nanosecond pulses at telecom wavelength $(1550 \mathrm{~nm}) .{ }^{11,12}$ For such long pulses, the energy deposition leads so far to uncontrolled (non-uniform) material changes. However, these and other recent observations with pulses longer than those of our experiment ${ }^{35}$ encourage us to test the effect of picosecond pulses in the near future. This may allow to minimize the thermal affects and contain enough energy (compared to femtosecond pulses) for modification while maintaining the power below the critical power for self-focusing in silicon.

A strong technological motivation for this work comes from the controlled high-aspect ratio nanochannel machining demonstrations achieved in glass. ${ }^{10}$ If similar demonstrations could be achieved in silicon, it would open the way to a new efficient technique for the fabrication of through-silicon via (TSV) that are vertical electrical connection passing completely through a silicon wafer to create $3 \mathrm{D}$ packages and $3 \mathrm{D}$ integrated circuits. ${ }^{36}$ More generally, if the nature of modification could be controlled along Bessel filaments in silicon, 
one could also envision optical guiding, fluid transport, and electrical interconnect functionalities fabricated on a monolithic silicon platform which are bases to build advanced hybrid devices or a lab-on-a-chip.

\section{ACKNOWLEDGMENTS}

This research was funded by the French National Research Agency (ANR 2010-JCJC-913-01) and the CARNOT STAR Institute (ViaLASER project).

${ }^{1}$ R. R. Gattass and E. Mazur, "Femtosecond laser micromachining in transparent materials," Nat. Photonics 2, 219-225 (2008).

${ }^{2}$ A. H. Nejadmalayeri, P. R. Herman, J. Burghoff, M. Will, S. Nolte, and A. Tünnermann, "Inscription of optical waveguides in crystalline silicon by mid-infrared femtosecond laser pulses," Opt. Lett. 30, 964 (2005).

${ }^{3}$ V. V. Kononenko, V. V. Konov, and E. M. Dianov, "Delocalization of femtosecond radiation in silicon," Opt. Lett. 37, 3369-3371 (2012).

${ }^{4}$ S. Leyder, D. Grojo, P. Delaporte, W. Marine, M. Sentis, and O. Utéza, "Multiphoton absorption of $1.3 \mu \mathrm{m}$ wavelength femtosecond laser pulses focused inside $\mathrm{Si}$ and $\mathrm{SiO}_{2}$," Proc. SPIE 8770, 877004 (2013).

${ }^{5}$ A. Mouskeftaras, A. V. Rode, R. Clady, M. Sentis, O. Utéza, and D. Grojo, "Self-limited underdense microplasmas in bulk silicon induced by ultrashort laser pulses," Appl. Phys. Lett. 105, 191103 (2014).

${ }^{6}$ A. D. Bristow, N. Rotenberg, and H. M. van Driel, "Two-photon absorption and Kerr coefficients of silicon for 8502200 nm," Appl. Phys. Lett. 90, 191104 (2007).

${ }^{7}$ D. Grojo, S. Leyder, P. Delaporte, W. Marine, M. Sentis, and O. Utéza, "Long-wavelength multiphoton ionization inside band-gap solids," Phys. Rev. B 88, 195135 (2013).

${ }^{8}$ A. Couairon and A. Mysyrowicz, "Femtosecond filamentation in transparent media," Phys. Rep. 441, 47-189 (2007).

${ }^{9}$ D. Faccio, E. Rubino, A. Lotti, A. Couairon, A. Dubietis, G. Tamošauskas, D. G. Papazoglou, and S. Tzortzakis, "Nonlinear light-matter interaction with femtosecond high-angle Bessel beams," Phys. Rev. A 85, 033829 (2012).

${ }^{10}$ M. K. Bhuyan, F. Courvoisier, P. A. Lacourt, M. Jacquot, R. Salut, L. Furfaro, and J. M. Dudley, "High aspect ratio nanochannel machining using single shot femtosecond Bessel beams," Appl. Phys. Lett. 97, 081102 (2010).

${ }^{11}$ P. C. Verburg, G. R. B. E. Römer, and A. J. Huis in t Veld, "Two-photoninduced internal modification of silicon by erbium-doped fiber laser," Opt. Express 22, 21958 (2014)

${ }^{12}$ O. Tokel, A. Turnali, I. Pavlov, S. Tozburun, I. Akca, and F. Ilday, "Laserwriting in silicon for 3D information processing," e-print arXiv: $1409.2827 \mathrm{v} 1$

${ }^{13}$ M. Mori, Y. Shimotsuma, T. Sei, M. Sakakura, K. Miura, and H. Udono, "Tailoring thermoelectric properties of nanostructured crystal silicon fabricated by infrared femtosecond laser direct writing," Phys. Status Solidi A 212, 715-721 (2015)

${ }^{14}$ R. M. Herman and T. A. Wiggins, "Production and uses of diffractionless beams," J. Opt. Soc. Am. A 8, 932 (1991).

${ }^{15}$ Z. Bomzon, M. Gu, and J. Shamir, "Angular momentum and geometrical phases in tight-focused circularly polarized plane waves," Appl. Phys. Lett. 89, 241104 (2006)

${ }^{16}$ S. Leyder, D. Grojo, P. Delaporte, W. Marine, M. Sentis, and O. Utéza, "Non-linear absorption of focused femtosecond laser pulses at $1.3 \mu \mathrm{m}$ inside silicon: Independence on doping concentration," Appl. Surf. Sci. 278, 13-18 (2013).
${ }^{17}$ J. M. Liu, "Simple technique for measurements of pulsed Gaussian-beam spot sizes," Opt. Lett. 7, 196 (1982).

${ }^{18}$ C. Hnatovsky, V. Shvedov, W. Krolikowski, and A. Rode, "Revealing local field structure of focused ultrashort pulses," Phys. Rev. Lett. 106, 123901 (2011).

${ }^{19}$ D. Grojo, M. Gertsvolf, H. Jean-Ruel, S. Lei, L. Ramunno, D. M. Rayner, and P. B. Corkum, "Self-controlled formation of microlenses by optical breakdown inside wide-band-gap materials," Appl. Phys. Lett. 93, 243118 (2008).

${ }^{20}$ A. Joglekar, H. Liu, G. Spooner, E. Meyhfer, G. Mourou, and A. Hunt, “A study of the deterministic character of optical damage by femtosecond laser pulses and applications to nanomachining," Appl. Phys. B: Lasers Opt. 77, 25-30 (2003).

${ }^{21}$ C. Hnatovsky, E. Simova, P. P. Rajeev, D. M. Rayner, P. B. Corkum, and R. S. Taylor, "Femtosecond laser writing of porous capillaries inside fused silica glass," Opt. Lett. 32, 1459-1461 (2007).

${ }^{22}$ E. G. Gamaly, B. Luther-Davies, L. Hallo, P. Nicolai, and V. T. Tikhonchuk, "Laser-matter interaction in the bulk of a transparent solid: Confined microexplosion and void formation," Phys. Rev. B 73, 214101 (2006)

${ }^{23}$ Q. Lin, J. Zhang, G. Piredda, R. W. Boyd, P. M. Fauchet, and G. P. Agrawal, "Dispersion of silicon nonlinearities in the near infrared region," Appl. Phys. Lett. 91, 021111 (2007).

${ }^{24}$ A. Dragomir, J. G. Mcinerney, and D. N. Nikogosyan, "Femtosecond measurements of two-photon absorption coefficients at $264 \mathrm{~nm}$ in glasses, crystals, and liquids," Appl. Opt. 41, 4365-4376 (2002).

${ }^{25}$ A. Dragomir, J. G. McInerney, D. N. Nikogosyan, and P. G. Kazansky, "Two-photon absorption properties of commercial fused silica and germanosilicate glass at $264 \mathrm{~nm}$, , Appl. Phys. Lett. 80, 1114 (2002).

${ }^{26}$ J. Amako, D. Sawaki, and E. Fujii, "Microstructuring transparent materials by use of nondiffracting ultrashort pulse beams generated by diffractive optics,” J. Opt. Soc. Am. B 20, 2562 (2003).

${ }^{27}$ M. K. Bhuyan, P. K. Velpula, J. P. Colombier, T. Olivier, N. Faure, and R. Stoian, "Single-shot high aspect ratio bulk nanostructuring of fused silica using chirp-controlled ultrafast laser Bessel beams," Appl. Phys. Lett. 104, 021107 (2014).

${ }^{28}$ Y. Matsuoka, Y. Kizuka, and T. Inoue, "The characteristics of laser micro drilling using a Bessel beam,” Appl. Phys. A 84, 423-430 (2006).

${ }^{29}$ W.-J. Tsai, C.-J. Gu, C.-W. Cheng, and J.-B. Horng, "Internal modification for cutting transparent glass using femtosecond Bessel beams," Opt. Eng. 53, 051503 (2013)

${ }^{30} \mathrm{M}$. Duocastella and C. Arnold, "Bessel and annular beams for materials processing," Laser Photonics Rev. 6, 607-621 (2012).

${ }^{31}$ I. Bogatyrev, D. Grojo, P. Delaporte, S. Leyder, M. Sentis, W. Marine, and T. Itina, "Non-linear absorption of $1.3-\mu \mathrm{m}$ wavelength femtosecond laser pulses focused inside semiconductors: Finite difference time domaintwo temperature model combined computational study," J. Appl. Phys. 110, 103106 (2011).

${ }^{32}$ J. Bonse, S. Baudach, J. Krüger, W. Kautek, and M. Lenzner, "Femtosecond laser ablation of siliconmodification thresholds and morphology," Appl. Phys. A 74, 19-25 (2002).

${ }^{33}$ M. Dinu, F. Quochi, and H. Garcia, "Third-order nonlinearities in silicon at telecom wavelengths," Appl. Phys. Lett. 82, 2954 (2003).

${ }^{34}$ Handbook of Chemistry and Physics, 85 th ed., edited by D. Lide (CRC Press, 2003)

${ }^{35}$ Y. Ito, H. Sakashita, R. Suzuki, M. Uewada, K. Luong, and R. Tanabe, "Modification and machining on back surface of a silicon substrate by femtosecond laser pulses at $1552 \mathrm{~nm}$," J. Laser Micro/Nanoeng. 9, 98-102 (2014).

${ }^{36} \mathrm{~B}$. Wu, A. Kumar, and S. Pamarthy, "High aspect ratio silicon etch: A review,” J. Appl. Phys. 108, 051101 (2010). 